\title{
Influence relative de la morphologie cellulaire et de l'angle des microfibrilles sur l'anisotropie élastique tissulaire Longitudinale/Tangentielle du bois sans défaut des résineux
}

\author{
Christophe GACHET ${ }^{\mathrm{a} *}$, Daniel GUITARD ${ }^{\mathrm{b}}$ \\ a CEA Le Ripault, Laboratoire Carbone et Composites, LR/DMAT/SR2C/LCC, B.P 16, 37260 Monts, France \\ b TREFLE - ENSAM, UMR CNRS 8508, Esplanade des Arts et Métiers, 33405 Talence Cedex, France
}

(Reçu le 17 septembre 2004 ; accepté le 9 janvier 2006)

\begin{abstract}
Résumé - L'influence de l'angle des microfibrilles sur le rapport d'anisotropie élastique tissulaire Longitudinal/Tangentiel (L/T) des résineux est abordée au travers d'une modélisation multi échelles. L'anisotropie élastique d'un tissu ligneux $\left(\mathrm{E}_{\mathrm{L}}^{*} / \mathrm{E}_{\mathrm{T}}^{*}\right)$ résulte pour une part d'un effet géométrique associé à la morphologie de la trachéide et d'autre part de l'anisotropie de la double paroi cellulaire gouvernée essentiellement par l'angle des microfibrilles $(\varphi)$ dans la sous couche pariétale $S_{2}$. Il est montré ici que l'anisotropie tissulaire d'un bois initial est principalement pilotée par la géométrie des arrangements de cellules conduisant à la flexion des parois. S'agissant d'un bois final, l'anisotropie tissulaire résulte principalement de la faible inclinaison des microfibrilles dans $S_{2}$.
\end{abstract}

anisotropie élastique / modélisation multi échelles / angle des microfibrilles

\begin{abstract}
Relative influence of cellular morphology and microfibrillar angle on Longitudinal/Tangential elastic anisotropy of softwood tissues. Microfibrillar angle (MFA) influence on elastic anisotropy Longitudinal/Tangential (L/T) of softwood wooden tissues is illustrated through a multi scale modelling. Elastic anisotropy results in the one hand from a geometric effect of cellular morphology and, in the other hand, from double cell wall anisotropy controlled by microfibrillar angle in $S_{2}$ sub layer. As a result, it is shown here that tissular anisotropy of early wood is mainly governed by the geometry of cellular network, whereas MFA is an essential parameter to explain the latewood tissue anisotropy.
\end{abstract}

elastic anisotropy /multi scale modelling / microfibrillar angle

\section{INTRODUCTION}

Deux particularités marquantes du comportement élastique du matériau bois sont, d'une part la très grande rigidité spécifique $\mathrm{E}_{\mathrm{L}} / \rho$ dans la direction longitudinale, et d'autre part la très forte anisotropie élastique $\mathrm{E}_{\mathrm{L}} / \mathrm{E}_{\mathrm{T}}$ (Tab. I). Dans le cas du résineux standard $[10,12]$, une valeur de $\mathrm{E}_{\mathrm{L}} / \mathrm{E}_{\mathrm{T}}$ proche de 20 peut en outre être considérée comme un minorant de l'anisotropie tissulaire $\left(\mathrm{E}_{\mathrm{L}}^{*} / \mathrm{E}_{\mathrm{T}}^{*}\right)$ du fait de l'existence d'une fraction volumique en rayons ligneux contribuant à l'augmentation de la rigidité radiale $\mathrm{E}_{\mathrm{R}}$ au détriment de la rigidité longitudinale $\mathrm{E}_{\mathrm{L}}$.

La plupart des propriétés mécanophysiques observées à l'échelle du bois massif (échelle macroscopique) résulte d'une imbrication à priori étroite de plusieurs niveaux d'hétérogénéité : échelle supramoléculaire (microfibrilles de cellulose, polymères amorphes), échelle pariétale (orientation des microfibrilles dans les sous couches), échelle de la trachéide (morphologie de la cellule), échelle du tissu cellulaire (différenciation en bois initial/bois final) et enfin échelle du cerne (présence de rayons ligneux).

Dans un travail antérieur [7, 12], mené sur la base d'une large analyse documentaire, les sources principales de variabilité intra arbre de l'anisotropie élastique macroscopique des essences résineuses ont été inventoriées. Les échelles de descriptions (ou « tiroirs » de modélisation) envisagées à l'issu de ce recensement ont été strictement limitées à l'essentiel : l'échelle de la sous couche pariétale, l'échelle de la double cloison cellulaire, l'échelle du tissu ligneux et l'échelle du cerne d'accroissement.

Une succession de modèles micro macro décrits auparavant [12] et appelés Passages, a donc été proposée afin d'assurer la transition entre les paramètres descriptifs de la microstructure des cellules ligneuses et le comportement élastique macroscopique

\footnotetext{
* Auteur pour correspondance : gachetchristophe2@yahoo.fr
} 


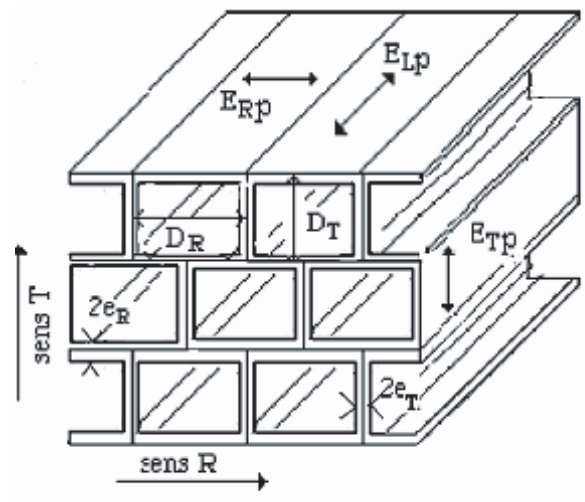

a)

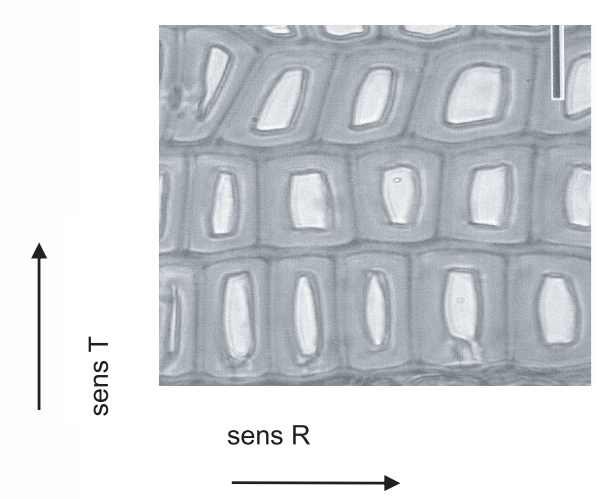

b)

Figure 1. (a) Structure poreuse retenue comme représentation schématique de tissu ligneux ; (b) coupe transversale d'épicéa colorée à la safranine et au bleu alcyan (grossissement 500, barre d'échelle 25 microns) [22].

Tableau I. Position du module spécifique et de l'anisotropie élastique macroscopique $\mathrm{E}_{\mathrm{L}} / \mathrm{E}_{\mathrm{T}}$ des «bois standards » en regard des mêmes caractéristiques de quelques matériaux industriels usuels.

\begin{tabular}{lcccc}
\hline & $\begin{array}{c}\text { Feuillu } \\
\text { standard }\end{array}$ & $\begin{array}{c}\text { Résineux } \\
\text { standard }\end{array}$ & Ver. / époxy & Epoxy \\
\hline $\mathrm{E}_{\mathrm{L}} / \rho(\mathrm{J} / \mathrm{kg})$ & 22200 & 29100 & 19000 & 2370 \\
$\mathrm{E}_{\mathrm{L}} / \mathrm{E}_{\mathrm{T}}$ & 14,0 & 20,6 & 2,75 & 1 \\
\hline
\end{tabular}

du matériau, notamment l'anisotropie Longitudinale/Tangentielle $\left(\mathrm{E}_{\mathrm{L}}^{*} / \mathrm{E}_{\mathrm{T}}^{*}\right)$ des tissus constitutifs (bois initial, bois final et rayon ligneux). On précise ici que la vocation principale de cette pyramide de modélisation est moins de permettre une estimation chiffrée précise des propriétés globales à partir des propriétés des constituants, que de faire clairement sentir à travers des schématisations simples mais réalistes, les sens de variation des grandeurs macroscopiques en regard des variables structurales retenues.

Le Passage 1 décrit, via 5 paramètres, l'élasticité de la sous couche pariétale considérée comme une matrice amorphe isotrope $\left(\mathrm{E}^{\mathrm{m}}, v^{\mathrm{m}}\right.$; respectivement module élastique et coefficient de Poisson de la matrice) de lignines et d'hémicelluloses renforcée par une fraction volumique $(\mathrm{V})$ de microfibrilles cellulosiques isotropes $\left(\mathrm{E}^{\mathrm{f}}, \mathrm{v}^{\mathrm{f}}\right.$ : module élastique et coefficient de Poisson des microfibrilles), quasi cristallines, et orientées.

Le Passage 2 conduit à des estimations de l'élasticité longitudinale $\left(\mathrm{E}_{\mathrm{Lp}}\right)$ et tangentielle $\left(\mathrm{E}_{\mathrm{Tp}}\right)$ du bicouche que constitue la double paroi de deux trachéides adjacentes, éventuellement assimilée (dans le cas de modèles multicouches) à un stratifié de $\mathrm{p}$ sous couches anisotropes $\left(\mathrm{S}_{1}, \mathrm{~S}_{2}, \mathrm{~S}_{3}\right)$ orientées. À cette étape de description, chacun des plis $\mathrm{p}$ est identifié par son épaisseur $\left(\mathrm{e}^{\mathrm{p}}\right)$ et l'angle des microfibrilles $\left(\varphi^{\mathrm{p}}\right)$.

Le Passage 3 fournit les modules élastiques tissulaires $E_{L}^{*}$, $\mathrm{E}_{\mathrm{R}}^{*}, \mathrm{E}_{\mathrm{T}}^{*}$ du solide homogène équivalent à une structure poreuse périodique à motifs rectangulaires (ensemble de trachéides localement identiques) compte tenu de la morphologie $\left(\mathrm{D}_{\mathrm{R}}, \mathrm{e}_{\mathrm{R}}\right.$, $\mathrm{D}_{\mathrm{T}}, \mathrm{e}_{\mathrm{T}}$ : respectivement diamètres et épaisseurs de parois radiales et tangentielles) et des arrangements cellulaires du type ali- gnement radial et distribution en quinconce tangentiel (Figs. 1a et $1 \mathrm{~b})$. Les hypothèses mécaniques simplificatrices utilisées pour la construction de ce Passage 3 , pour certaines évoquées dans d'autres travaux $[8,15,21]$, ont été précédemment présentées et discutées [12]. On rappelle ici que des trachéides adjacentes sont supposées parfaitement adhérentes (condition de non -glissement sur la lamelle mitoyenne), que les efforts appliqués suivant la direction radiale se transmettent par les parois radiales supposées en prolongement les unes des autres, et que l'application d'un chargement dans la direction tangentielle conduit à un comportement élastique pariétal de type « poutre en flexion trois points».

Chacune des étapes intermédiaires de la modélisation gigogne mise en place ici est susceptible d'être traitée par des modèles plus ou moins sophistiqués; pour chacun des niveaux d'hétérogénéité du matériau, de nombreux raffinements de modélisation sont donc envisageables. Néanmoins, l'objectif fixé ici s'inscrit plus dans la mise en place d'une approche globale déterministe, menée par l'intuition des phénomènes cruciaux gouvernant l'anisotropie élastique du matériau, que dans le cadre d'une étude numérique, sans doute histologiquement plus réaliste, associant techniques d'analyse d'image à des calculs d'homogénéisation. Une première alternative de sophistication des sous étapes de description est cependant prise en compte dans notre travail. Ainsi, à l'échelle ultra structurale, un modèle « Squelette » [11], substituant aux Passages 1 et 2 ci-dessus un Passage 1-2, assure la transition directe des macromolécules constitutives aux propriétés élastiques $\left(\mathrm{E}_{\mathrm{Lp}}, \mathrm{E}_{\mathrm{Tp}}\right) \mathrm{d}$ 'une double paroi à isotropie transverse, ceci en privilégiant les propriétés de la sous couche $S_{2}$ (histologiquement et mécaniquement 
prépondérante). Pour décrire mécaniquement cette double paroi, un jeu de 6 paramètres micro mécaniques particularisés $\left(E^{\mathrm{m}}, v^{\mathrm{m}}, \mathrm{E}^{\mathrm{f}}, \mathrm{v}^{\mathrm{f}}, \mathrm{V}\right.$ et $\left.\varphi\right)$ est proposé à partir de données cytologiques. Le modèle Squelette, utilisé dans la suite de la discussion, est basé sur le concept d'une cellule virtuelle dont la paroi est renforcée par un treillis de microfibrilles constitué de deux nappes antisymétriques d'orientation microfibrillaire $(+\varphi)$ et $(-\varphi)$. Sans doute «biologiquement» moins représentatif que les modèles multicouches classiques, il présente l'avantage « mécanique »- comparativement à ces mêmes stratifiés - de prendre directement en compte la restriction de cisaillement [3, 18] qu'implique le Passage 2 de la trachéide au tissu ligneux. Assumant, à l'échelle tissulaire, la rigidification induite par le blocage de l'effet Poisson [11] ; le Squelette se présente donc, de manière originale, plus comme un modèle de tissu ligneux homogène (Passage 1-2) que comme un modèle de cellule isolée de son environnement mécanique.

Le coefficient d'anisotropie élastique tissulaire $\left(\mathrm{E}_{\mathrm{L}}^{*} / \mathrm{E}_{\mathrm{T}}^{*}\right)$, produit des deux autres coefficients d'anisotropie élastique Radiale/Tangentielle $\left(\mathrm{E}_{\mathrm{R}}^{*} / \mathrm{E}_{\mathrm{T}}^{*}\right)$ et Longitudinale/Radiale $\left(\mathrm{E}_{\mathrm{L}}^{*} / \mathrm{E}_{\mathrm{R}}^{*}\right)$, s'écrit donc, au travers de l'approche micro-macro, sous la forme de trois termes multiplicatifs $a_{R T}, a_{L R}$ et $a_{L T p}$ directement exprimés en fonction des paramètres structuraux et ultra structuraux sélectionnés :

$$
\frac{E_{L}^{*}}{E_{T}^{*}}=\frac{E_{R}^{*}}{E_{T}^{*}} \times \frac{E_{L}^{*}}{E_{R}^{*}}=a_{R T} \cdot a_{L R} \cdot a_{L T p}
$$

En posant

$$
\begin{gathered}
a_{R T}=\frac{E_{R}^{*}}{E_{T}^{*}}=\left[1+\frac{1}{64} \frac{e_{T}}{D_{T}} \frac{\left(D_{R}-2 e_{T}\right)^{3}}{e_{R}^{3}}\right] \\
\text { et } a_{L T p}=\frac{E_{L R}}{E_{T p}}=\left[1+\frac{e_{R}}{e_{T}} \frac{D_{R}}{D_{T}}-2 \frac{e_{R}}{D_{T}}\right] \\
=\left[\frac{(1-V) \frac{\left(1-v^{m}\right)}{\left(1+v^{m}\right)\left(1-2 v^{m}\right)} E^{m}+V\left(1-\sin ^{4}(\varphi) E^{f}\right)}{(1-V) \frac{\left(1-v^{m}\right)}{\left(1+v^{m}\right)\left(1-2 v^{m}\right)} E^{m}+V\left(1-\cos ^{4}(\varphi) E^{f}\right)}\right]
\end{gathered}
$$

Dans la suite de l'étude, en accord avec certaines des sources bibliographiques $[4,5,16,17]$, aucune différence ne sera faite entre les épaisseurs pariétales radiale $\mathrm{e}_{\mathrm{R}}$ et tangentielle $\mathrm{e}_{\mathrm{T}}$. On adoptera donc $\mathrm{e}_{\mathrm{R}}=\mathrm{e}_{\mathrm{T}}=\mathrm{e}$.

D'autre part, la morphologie cellulaire, réduite à celle d'un tube creux de section droite rectangulaire (Fig. 1), sera paramétrée par deux grandeurs adimensionnelles $\delta$ et $\Gamma$, construites sur la base du diamètre cellulaire tangentiel $\mathrm{D}_{\mathrm{T}}$, noté $\mathrm{D}$; cette grandeur est en effet généralement plus stable que le diamètre radial $\mathrm{D}_{\mathrm{R}}$, car générée au cours de la croissance aux dimensions de la cellule mère de l'assise cambiale :

$$
\begin{aligned}
& \delta=\frac{D_{R}}{D_{T}}=\frac{D_{R}}{D}: \text { paramètre d'expansion radiale cellulaire, } \\
& \Gamma=\frac{2 \mathrm{e}}{\mathrm{D}}: \text { rapport de forme cellulaire. }
\end{aligned}
$$

En conséquence, la relation (1) est naturellement décomposée en un coefficient $\mathrm{E}_{\mathrm{R}}^{*} / \mathrm{E}_{\mathrm{T}}^{*}$, caractérisant l'anisotropie élastique dans le plan transverse $(\vec{R}, \vec{T})$ du tissu ligneux,

$$
\frac{\mathrm{E}_{\mathrm{R}}^{*}}{\mathrm{E}_{\mathrm{T}}^{*}}=\mathrm{a}_{\mathrm{RT}}=\left[1-\frac{\Gamma}{16}\left(1-\frac{\delta}{\Gamma}\right)^{3}\right]
$$

et en un coefficient $\mathrm{E}_{\mathrm{L}}^{*} / \mathrm{E}_{\mathrm{R}}^{*}$, caractéristique de l'anisotropie élastique du tissu ligneux dans le plan longitudinal / radial $(\overrightarrow{\mathrm{L}}$, $\overrightarrow{\mathrm{R}}$ ), exprimé en (3).

$$
\begin{aligned}
\frac{\mathrm{E}_{\mathrm{L}}^{*}}{\mathrm{E}_{\mathrm{R}}^{*}} & =[1+\delta-\Gamma] \\
& \times\left[\frac{(1-V) \frac{\left(1-v^{\mathrm{m}}\right)}{\left(1+v^{\mathrm{m}}\right)\left(1-2 v^{\mathrm{m}}\right)} \mathrm{E}^{\mathrm{m}}+\mathrm{V}\left(1-\sin ^{4}(\varphi) \mathrm{E}^{\mathrm{f}}\right)}{(1-V) \frac{\left(1-v^{\mathrm{m}}\right)}{\left(1+v^{\mathrm{m}}\right)\left(1-2 v^{\mathrm{m}}\right)} \mathrm{E}^{\mathrm{m}}+\mathrm{V}\left(1-\cos ^{4}(\varphi) \mathrm{E}^{\mathrm{f}}\right)}\right]
\end{aligned}
$$

En référence à la microstructure (morphologie et organisation des trachéides dans un tissu ligneux) et à l'ultra structure (orientation $\varphi$ des microfibrilles dans la sous couche $S_{2}$ ), il s'agit ici d'interpréter chacun des termes des expressions (2) et (3) et de chiffrer leur influence relative sur le rapport d'anisotropie élastique $\left(\mathrm{E}_{\mathrm{L}}^{*} / \mathrm{E}_{\mathrm{T}}^{*}\right)$.

- Le produit des deux premiers termes de (1) représentent l'anisotropie géométrique dite «tissulaire » notée par la suite $a_{L T}$ (relation (4)). Celui-ci prend en compte au Passage 3, la morphologie cellulaire (i.e. géométrie et arrangements des motifs schématisés Fig. 1) caractérisée par les quatre paramètres géométriques biologiquement non indépendants $\left(\mathrm{D}_{\mathrm{R}}, \mathrm{D}_{\mathrm{T}}\right.$, $\left.\mathrm{e}_{\mathrm{R}}, \mathrm{e}_{\mathrm{T}}\right)$.

$\mathrm{a}_{\mathrm{LT}}=\mathrm{a}_{\mathrm{RT}} \cdot \mathrm{a}_{\mathrm{LR}}=\left[1-\frac{\Gamma}{16}\left(1-\frac{\delta}{\Gamma}\right)^{3}\right][1+\delta-\Gamma]$.

Remarque : On notera que sous l'hypothèse d'une masse volumique de la matière ligneuse constante $\left(\rho_{\mathrm{m}}=1510 \mathrm{~kg} / \mathrm{m}^{3}\right)$, la prise en compte de cette microstructure $\left(\mathrm{D}_{\mathrm{R}}, \mathrm{D}_{\mathrm{T}}, \mathrm{e}_{\mathrm{R}}, \mathrm{e}_{\mathrm{T}}\right)$ permet de relier (5) la masse volumique $\rho^{*}$ tissulaire à la fraction volumique de vide (i.e. à la porosité $\mathrm{P}_{0}$ ) selon :

$$
\begin{aligned}
\rho^{*}=\rho_{m}\left(1-P_{0}\right) & =2 \rho_{m}\left[\frac{\mathrm{e}_{\mathrm{T}}}{\mathrm{D}_{\mathrm{R}}}+\frac{\mathrm{e}_{\mathrm{R}}}{\mathrm{D}_{\mathrm{T}}}-2 \frac{\mathrm{e}_{\mathrm{T}} \mathrm{e}_{\mathrm{R}}}{\mathrm{D}_{\mathrm{R}} \mathrm{D}_{\mathrm{T}}}\right] \\
& =\rho_{m} \Gamma\left[1+\frac{1}{\delta}-\frac{\Gamma}{\delta}\right] .
\end{aligned}
$$

- Le dernier terme de l'expression (1), noté a ${ }_{\text {LTp }}$, caractérise l'anisotropie de la double paroi à isotropie ${ }^{\mathrm{LTp}}$ 'ransverse du modèle Squelette, laquelle est pilotée très majoritairement par l'angle des microfibrilles $(\varphi)$ dans la sous couche $S_{2}$ (Passages $1,2$ et $1-2,[7])$.

\section{INFLUENCE DE LA STRUCTURE ALVÉOLÉE SUR L'ANISOTROPIE GÉOMÉTRIQUE TISSULAIRE}

Il s'agit ici de chiffrer l'incidence de modifications (survenant notamment au cours de la différenciation bois initial - bois final) de la masse volumique tissulaire et de la disposition des 


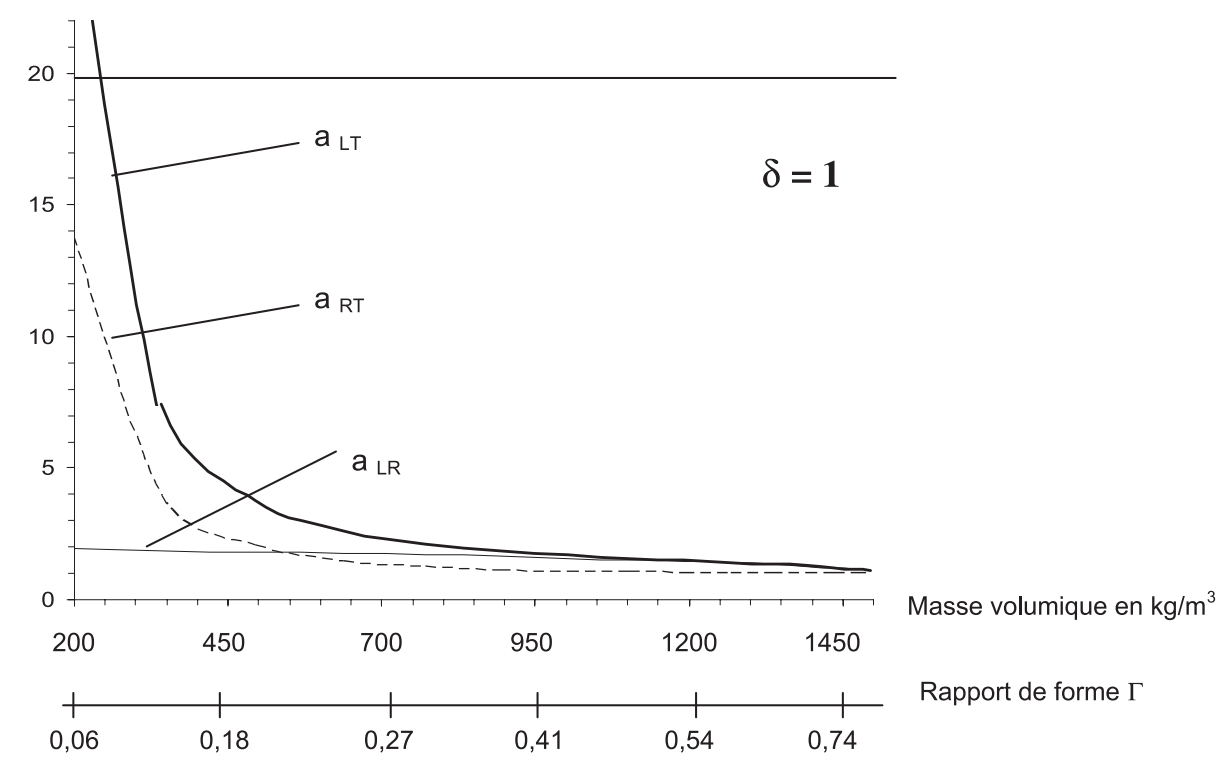

Figure 2. Évolutions des rapports d'anisotropie géométrique tissulaire avec la masse volumique et le rapport de forme dans le cas de cellules carrées.

parois (arrangements des trachéides) sur l'anisotropie géométrique du tissu homogène équivalent. Les applications numériques sont conduites à partir d'observations expérimentales de la microstructure rapportées dans la bibliographie (épaisseurs pariétales et diamètres cellulaires, [7]).

\subsection{Contribution de la masse volumique à l'anisotropie géométrique tissulaire}

\subsubsection{Cas de cellules carrées}

Sous l'hypothèse de diamètres radial $\mathrm{D}_{\mathrm{R}}$ et tangentiel $\mathrm{D}_{\mathrm{T}}$ identiques $\left(\mathrm{D}_{\mathrm{R}}=\mathrm{D}_{\mathrm{T}}=\mathrm{D}\right.$, i.e. $\left.\delta=1\right)$, le facteur géométrique d'anisotropie $\mathrm{a}_{\mathrm{LT}}$ défini en (4) est directement lié au rapport de forme cellulaire $\Gamma$ par la relation suivante :

$$
\mathrm{a}_{\mathrm{LT}}=[2-\Gamma]\left[1-\frac{\Gamma}{16}\left(\frac{1}{\Gamma}\right)^{3}\right]=[2-\Gamma]\left(1-\mathrm{a}_{\mathrm{F}}\right) .
$$

En posant :

$$
\mathrm{a}_{\mathrm{F}}=\frac{\Gamma}{16}\left(1-\frac{1}{\Gamma}\right)^{3} .
$$

Le premier terme de $\mathrm{a}_{\mathrm{LT}}$ (relation (6)), fonction de $\Gamma$, caractérise l'anisotropie élastique tissulaire $\mathrm{a}_{\mathrm{LR}}$, participant à l'anisotropie $\left(\mathrm{E}_{\mathrm{L}}^{*} / \mathrm{E}_{\mathrm{T}}^{*}\right)$ et imputable à la fois â la structure alvéolée et à l'alignement pariétal des trachéides carrées dans le sens $\mathrm{R}$. L'expression $\left(1+\mathrm{a}_{\mathrm{F}}\right)$, fonction, elle aussi de $\Gamma$, représente quand à elle la part d'anisotropie élastique tissulaire $a_{\mathrm{RT}}$ impliquée dans $\mathrm{a}_{\mathrm{LT}}$ et résultant de la mise en flexion de la double cloison cellulaire (arrangements en quinconces dans le sens T, [12]).

Sous les conditions géométriques précitées, la masse volumique $\rho *$ s'exprimera alors directement en fonction du rapport de forme $\Gamma$ :

$$
\rho^{*}=\rho_{\mathrm{m}} \Gamma[2-\Gamma] .
$$

Sur la figure 2 sont reportées et illustrées, pour des cellules carrées, les valeurs prises par les facteurs géométriques d'anisotropie Longitudinale/Tangentielle a , Longitudinale/Radiale $\mathrm{a}_{\mathrm{LR}}$ et Radiale/Tangentielle $\mathrm{a}_{\mathrm{RT}}$ en fonction de la masse volumique tissulaire $\rho *$ et du rapport de forme $\Gamma$. La plage d'évolution choisie pour $\rho^{*}$ correspond à celle théoriquement observée lors de la transition d'un tissu de bois initial peu dense $\left(\rho^{*}=200 \mathrm{~kg} / \mathrm{m}^{3}\right)$ à un tissu présentant une masse volumique proche de celle de la matière ligneuse $\left(\rho *=\rho_{m}\right)$.

On constate en premier lieu (Fig. 2) que l'augmentation de la masse volumique tissulaire $\rho *$ (i.e. augmentation du rapport de forme $\Gamma$ ) entraîne une diminution sensible des facteurs d'anisotropie $\mathrm{a}_{\mathrm{LT}}$ et $\mathrm{a}_{\mathrm{RT}}$. La décroissance du terme $\mathrm{a}_{\mathrm{RT}}$ (et donc de $a_{F}$ ) avec $\rho *$ traduit en effet une rigidification des parois de cellules sollicitées en flexion, allant jusqu'à conduire, pour des masses volumiques supérieures à $900 \mathrm{~kg} / \mathrm{m}^{3}$ (tissu de bois de réaction ou de bois final dense), à l'isotropie transverse tissulaire (i.e. $\mathrm{a}_{\mathrm{RT}}=1$ ).

La figure 2 montre par ailleurs que des valeurs de $a_{\text {LT }}$ proches de 20 ne sont atteintes que pour des masses volumiques inférieures à $250 \mathrm{~kg} / \mathrm{m}^{3}$. En particulier, avec $\rho^{*}=450 \mathrm{~kg} / \mathrm{m}^{3}$ (i.e. $\Gamma=0.18$ ), un tissu isolé de résineux standard ne présente qu'un facteur d'anisotropie élastique $a_{\text {t }}$ de l'ordre de 4 , bien inférieur à celui attendu sur un tissu de bois normal (de l'ordre de 20). En conséquence, la variable $\Gamma$ ne sera un acteur significatif de l'anisotropie Longitudinale/Tangentielle que pour des structures poreuses de faible masse volumique (par exemple un tissu de bois initial peu dense).

On note enfin la faible sensibilité du coefficient d'anisotropie géométrique $\mathrm{a}_{L R}$ aux variations du facteur de forme $\Gamma$ (i.e. aux variations de $\rho *$ ). Dans le cadre de motifs carrés, a en effet compris entre 1 (tissu à forte densité, i.e. bois de réaction ou bois final dense) et 2 (bois initial). 


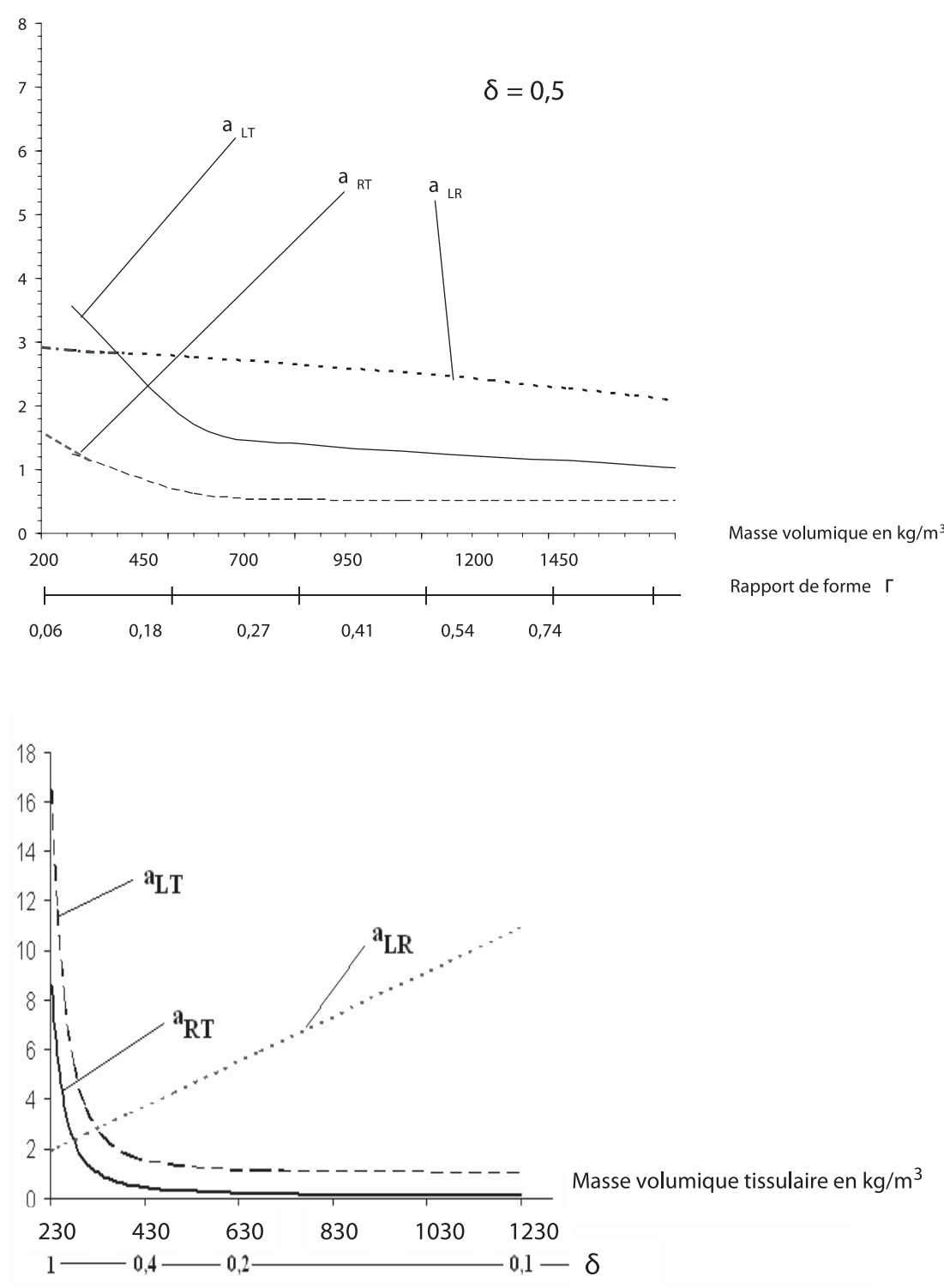

Figure 3. Évolutions des rapports d'anisotropie géométrique tissulaire avec la masse volumique tissulaire et le rapport de forme pour des cellules rectangulaires.

Figure 4. Évolutions des rapports d'anisotropie élastique avec l'expansion radiale et la masse volumique tissulaire.

\subsubsection{Cas des cellules rectangulaires}

La figure 3 illustre, à titre de complément de l'étude analytique précédente, les valeurs prises par les différents facteurs d'anisotropie géométrique en fonction de la masse volumique tissulaire d'une structure poreuse alvéolée à motifs rectangulaires $(\delta=0.5)$. Si les évolutions des différents facteurs d'anisotropie avec la masse volumique demeurent identiques à celles relevées précédemment pour des trachéides carrées (Sect. 2.1.1), on remarque que la diminution de la valeur assignée au paramètre $\delta$ se traduit par une augmentation du facteur d' anisotropie géométrique Longitudinale/Radiale $\mathrm{a}_{\mathrm{LR}}$. Celui-ci passe ainsi d'une valeur de 2 pour des cellules carrées à 3 dans le cas de cellules rectangulaires. Ceci s'explique par la grande sensibilité du module élastique longitudinal (plus importante que celle du module élastique radial) à une augmentation de la masse volumique tissulaire.

\subsection{Contribution des arrangements cellulaires à l'anisotropie élastique tissulaire dans le plan transverse}

En associant microphotographies de plan ligneux et relevés microdensitométriques, divers travaux $[4,5,16,17]$ ont montré que la transition morphologique d'une trachéide de bois initial à une trachéide de bois final s'opère à diamètre tangentiel $\left(\mathrm{D}_{\mathrm{T}}\right)$ constant. Les mêmes comparaisons histologiques ont en outre révélé que, durant la saison de végétation, cette différenciation s'accompagne surtout d'une diminution nette de diamètre radial $\left(\mathrm{D}_{\mathrm{R}}\right)$, associée à une croissance moindre de l'épaisseur pariétale (e).

Sur la base de ces observations physiologiques, une discussion est à nouveau menée afin d'apprécier l'impact d'une expansion radiale cellulaire sur l'anisotropie géométrique de la structure périodique poreuse. La figure 4 illustre donc les modifications des coefficients d'anisotropie géométriques associées 


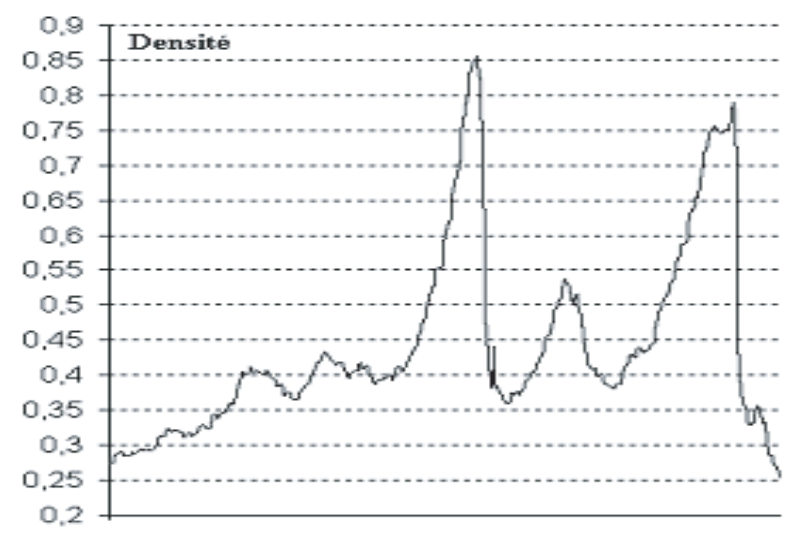

Figure 5. Profil micro densitométrique d'épicéa.

à une diminution du diamètre radial $\left(\mathrm{D}_{\mathrm{R}}\right)$ par rapport au diamètre tangentiel $\left(\mathrm{D}_{\mathrm{T}}\right)$. Les applications numériques sont conduites à partir d'un rapport de forme $\Gamma$ de 0.08 ce qui correspond pour des trachéides carrées à une masse volumique de $250 \mathrm{~kg} / \mathrm{m}^{3}$ observable sur des tissus de bois initial peu denses (Fig. 5, [7]).

Dans ces conditions la figure 4 permet de constater que pour des valeurs de $D_{R}$ décroissantes (diminution de $\delta$ ) la masse volumique tissulaire augmente (d'où une diminution de la porosité), et que $a_{L T}$ et $a_{L R}$ évoluent en sens opposé. Ceci se traduit par un facteur d'anisotropie géométrique $a_{\mathrm{RT}}$ qui devient inférieur à 1 , avec pour conséquence majeure une inversion de l'anisotropie élastique tissulaire dans le plan transverse. Cette inversion de la relation d'ordre entre le module élastique tissulaire radial $\mathrm{E}_{\mathrm{R}}^{*}$ et le module élastique tissulaire tangentiel $\mathrm{E}_{\mathrm{T}}^{*}\left(\mathrm{a}_{\mathrm{RT}} \leq 1\right.$ pour $\left.\delta=0,33\right)$ est imputable au renforcement tangentiel cellulaire qu'induit la décroissance de $\mathrm{D}_{\mathrm{R}}$ (augmentation via $\delta$ de la proportion de la phase solide sollicitée en traction/ compression et diminution de la portion de paroi soumise à la flexion (relation (9), [12]).

$$
\mathrm{E}_{\mathrm{T}}^{*}=\frac{\Gamma}{\delta} \frac{1}{\left[1+\frac{\Gamma}{4}\left(\frac{\delta}{\Gamma}-1\right)^{3}\right]} \mathrm{E}_{\mathrm{Tp}} .
$$

\section{CONTRIBUTION DE L'ANGLE DES MICROFIBRILLES À L'ANISOTROPIE ÉLASTIQUE TISSULAIRE : $\mathbf{a}_{\text {LTP }}$}

Les paragraphes précédents (Sects. 2.1 et 2.2) viennent de clairement montrer que des degrés d'anisotropie tissulaire $\left(\mathrm{E}_{\mathrm{L}}^{*} /\right.$ $\mathrm{E}_{\mathrm{T}}^{*}$ ) de l'ordre de 20 ne peuvent être entièrement expliqués par des modifications morphologiques envisagées aussi bien à l'échelle de la trachéide (épaisseurs et diamètres cellulaires) qu'à l'échelle du tissu ligneux (alignement radial et quinconce tangentiel).

La justification de tels niveaux d'anisotropie élastique doit donc être recherchée à une échelle d'hétérogénéité immédiate- ment inférieure ; l'échelle de la double cloison (Passage 1-2) dont l'élasticité est principalement gouvernée [7] par l'orientation des microfibrilles.

Depuis longtemps [1, 13] ou plus récemment [2, 19, 24], l'inclinaison des microfibrilles $(\varphi)$ dans la sous couche $S_{2}$, est en effet considérée comme une donnée explicative cruciale de la variabilité inter et intra spécifique des propriétés élastiques macroscopiques $\left(\mathrm{E}_{\mathrm{L}}, \mathrm{E}_{\mathrm{R}}, \mathrm{E}_{\mathrm{T}}\right)$ du matériau bois.

\subsection{Sensibilité de l'anisotropie tissulaire à l'angle des microfibrilles}

En admettant, conformément à de nombreuses sources bibliographiques $[1,3,13,15,18]$, que la matière ligneuse peut être assimilée à un milieu bi phasique constitué, en proportion égales (i.e. V fixée à 0.5 ), d'une matrice amorphe renforcée par des microfibrilles de celluloses quasi cristallines, le rapport $a_{L T p}=\frac{E_{L p}}{E_{T p}}$ présenté en (1) devient :

$$
a_{L T p}=\frac{\frac{\left(1-v^{m}\right)}{\left(1+v^{m}\right)\left(1-2 v^{m}\right)} E^{m}+\left(1-\sin ^{4}(\varphi)\right) E^{f}}{\frac{\left(1-v^{m}\right)}{\left(1+v^{m}\right)\left(1-2 v^{m}\right)} E^{m}+\left(1-\cos ^{4}(\varphi)\right) E^{f}}
$$

Dans ces conditions, la valeur de la dérivée (11) du produit $\left(\mathrm{a}_{\mathrm{LT}} \cdot \mathrm{a}_{\mathrm{LTp}}\right)$ rend compte de la sensibilité de l'anisotropie tissulaire $\left(\mathrm{E}_{\mathrm{L}}^{*} / \mathrm{E}_{\mathrm{T}}^{*}\right)$ à l'angle des microfibrilles $(\varphi)$ dans la sous couche $S_{2}$ :

$$
\begin{aligned}
& \frac{d \frac{E_{L}^{*}}{E_{T}^{*}}}{d \varphi}=a_{L T} \frac{d}{d \varphi}\left[\frac{A \cdot E^{m}+\left(1-\sin ^{4}(\varphi)\right) E^{f}}{A \cdot E^{m}+\left(1-\cos ^{4}(\varphi)\right) E^{f}}\right] \\
& =-a_{L T}\left[\frac{4 \cos (\varphi) \sin (\varphi) \mathrm{E}^{\mathrm{f}}}{\left[\mathrm{AE} \mathrm{E}^{\mathrm{m}}+\left(1-\cos ^{4}(\varphi)\right) \mathrm{E}^{\mathrm{f}}\right]^{2}}\right. \\
& \left.\times\left\{\mathrm{AE}^{\mathrm{m}}+\mathrm{E}^{\mathrm{f}}\left(1-\sin ^{2}(\varphi) \cos ^{2}(\varphi)\right)\right\}\right] \text {. }
\end{aligned}
$$

En ayant posé ;

$$
A=\frac{\left(1-v^{m}\right)}{\left(1+v^{m}\right)\left(1-2 v^{m}\right)} .
$$

Compte tenu des valeurs (Tab. II) classiquement assignées aux paramètres micromécaniques $\left(E^{\mathrm{m}}, v^{\mathrm{m}}, \mathrm{E}^{\mathrm{f}}, \mathrm{v}^{\mathrm{f}}\right)$, il est alors possible prendre la mesure de la sensibilité de l'anisotropie $\left(\mathrm{E}_{\mathrm{L}}^{*} / \mathrm{E}_{\mathrm{T}}^{*}\right)$ à l'inclinaison des microfibrilles $(\varphi)$ en examinant les valeurs prises par la fonction sensibilité $S$ définie en (12) :

$$
\begin{aligned}
S(\varphi) & =-\frac{4 \cos (\varphi) \sin (\varphi) \mathrm{E}^{\mathrm{f}}}{\left[\mathrm{AE}^{\mathrm{m}}+\left(1-\cos ^{4}(\varphi)\right) \mathrm{E}^{\mathrm{f}}\right]^{2}} \\
& \times\left\{\mathrm{AE}^{\mathrm{m}}+\mathrm{E}^{\mathrm{f}}\left(1-\sin ^{2}(\varphi) \cos ^{2}(\varphi)\right)\right\}
\end{aligned}
$$


Tableau II. Valeurs attribuées aux paramètres micromécaniques du modèle Squelette.

\begin{tabular}{lcc}
\hline & Sous couche S2 & Références \\
\hline $\mathrm{E}^{\mathrm{f}}(\mathrm{GPa})$ : Module d'Young des microfibrilles & $62 \leq \mathrm{Ef} \leq 137$ & {$[6,7,12,23]$} \\
$v^{\mathrm{f}}:$ Coefficient de Poisson des microfibrilles & 0.1 & {$[18,20]$} \\
$\mathrm{V}(\%):$ Fraction volumique en microfibrilles cristallines & 50 & {$[2]$} \\
$\mathrm{E}^{\mathrm{m}}(\mathrm{GPa}):$ Module d'Young de la matrice & 2 & {$[1,3]$} \\
$v^{\mathrm{m}}:$ Coefficient de Poisson de la matrice & 0.3 & {$[9,14]$} \\
$\varphi$ (degrés): Angle des microfibrilles & $5 \leq \varphi \leq 45$ & {$[24]$} \\
\hline
\end{tabular}

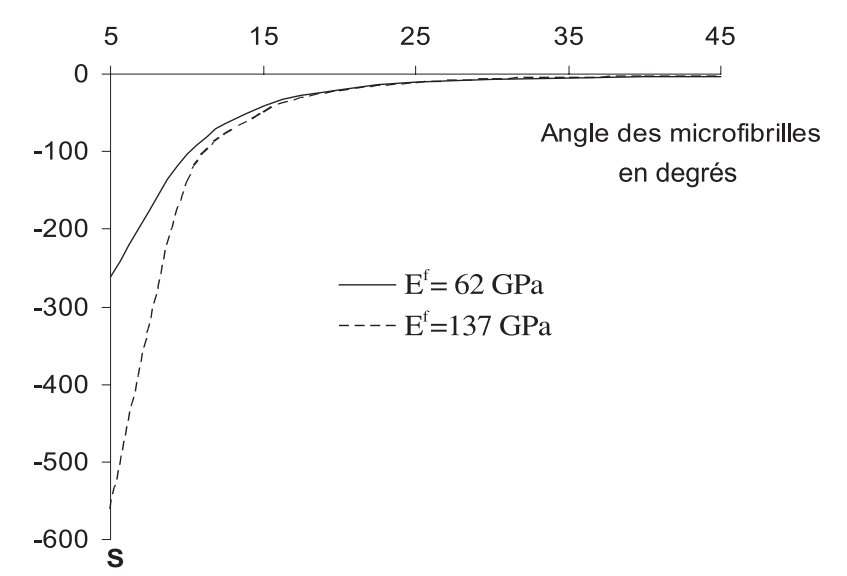

Figure 6. Relation d'ordre entre le facteur d'anisotropie pariétal a et le facteur géométrique d'anisotropie $\mathrm{a}_{\mathrm{LT}}$ en fonction de la masse volumique tissulaire et de l'expansion radiale cellulaire.

En conséquence, sont illustrées sur la figure 6 , les valeurs de la dérivée par rapport à $(\varphi)$ du rapport $\left(\mathrm{E}_{\mathrm{L}}^{*} / \mathrm{E}_{\mathrm{T}}^{*}\right)$, pour des inclinaisons de microfibrilles $(\varphi)$ couramment relevés sur des tissus de bois normal ( $\varphi$ allant de 5 à 10 degrés pour le bois final et de 15 à 35 degrés pour le bois initial) comme sur des tissus de bois de réaction ( $\varphi$ allant jusqu'à 45 degrés). La figure 6 montre que la fonction $\mathrm{S}$ est toujours négative et continûment décroissante dans l'intervalle $\left[5^{\circ}, 45^{\circ}\right]$. Quel que soit le module élastique longitudinal $\mathrm{E}^{\mathrm{f}}$ des fibres choisi pour alimenter le modèle, cette décroissance de $S$ avec $(\varphi)$ indique clairement que plus l'angle $(\varphi)$ est élevé, moins l'anisotropie élastique tissulaire $\left(\mathrm{E}_{\mathrm{L}}^{*} / \mathrm{E}_{\mathrm{T}}^{*}\right)$ en est dépendante. Des tissus de bois initial, de bois juvénile ou encore de bois de compression, particularisés à cette échelle par des inclinaisons $(\varphi)$ supérieures à 20 degrés [24], verront donc leur anisotropie $\left(\mathrm{E}_{\mathrm{L}}^{*} / \mathrm{E}_{\mathrm{T}}^{*}\right)$ moins sensible aux variations de l'angle $(\varphi)$ que des tissus de bois final à inclinaison microfibrillaire plus faible.

Cette dernière constatation est enfin à mettre en parallèle avec l'évolution du terme de flexion des parois $\mathrm{a}_{\mathrm{F}}$ (i.e. de $\mathrm{a}_{\mathrm{RT}}=$ $1+\mathrm{a}_{\mathrm{F}}$ ) en fonction de la masse volumique tissulaire. L'évolution de $\mathrm{a}_{\mathrm{RT}}$ avec la masse volumique rapportée sur les figures 2 et 3 montre ainsi, que la valeur du terme $a_{F}$ est d'autant plus importante que la masse volumique est faible. En conséquence, un tissu caractérisé à la fois par une masse volumique faible et un angle des microfibrilles ouvert (bois initial, bois juvénile) présentera donc une anisotropie tissulaire $\left(\mathrm{E}_{\mathrm{L}}^{*} / \mathrm{E}_{\mathrm{T}}^{*}\right)$ particulièrement sensible à la flexion des parois disposées en quinconces.

\subsection{Part d'anisotropie géométrique et part imputable à l'angle des microfibrilles}

La transition physiologique d'un tissu de bois initial à un tissu de bois final s'accompagne non seulement des modifications morphologiques évoquées précédemment (variation de $\Gamma$ et $\delta$, Sects. 2.1 et 2.2) mais également d'une diminution importante de l'angle des microfibrilles moyen dans la paroi cellulaire. Au cours de cette même différenciation annuelle, la proportion pariétale de sous couche $S_{2}$ augmente de plus très fortement. Compte tenu de ces remarques, une étude est à présent menée (à partir du modèle Squelette attribuant l'exclusivité du comportement élastique de la paroi à la sous couche $\mathrm{S}_{2}$ ) afin de dégager une hiérarchisation objective de l'influence de ces transformations biologiques sur l'anisotropie élastique tissulaire $\left(\mathrm{E}_{\mathrm{L}}^{*} / \mathrm{E}_{\mathrm{T}}^{*}\right)$ du matériau bois.

La figure 7 illustre de fait, pour 8 inclinaisons $(\varphi)$ et différentes masses volumiques tissulaires, les variations du rapport du facteur pariétal d'anisotropie $\mathrm{a}_{\mathrm{LTp}}$ sur le facteur géométrique d'anisotropie $\mathrm{a}_{\mathrm{LT}}$. Les applications numériques sont conduites à partir d'une cellule carrée de bois initial dont l'épaisseur de paroi et le diamètre sont fréquemment relevés [7] sur des microphotographies de plans ligneux $(\mathrm{D}=30 \mu \mathrm{m}$ et $\mathrm{e}=2 \mu \mathrm{m}$, i.e. $\Gamma=0.12$ ).

On remarque ainsi (Fig. 7) que quelle que soit la valeur de la masse volumique (et donc de $\Gamma$ ), des angles $(\varphi)$ compris entre 5 et 10 degrés conduisent à des valeurs de $\mathrm{a}_{\mathrm{L}}$ supérieures aux valeurs de $a_{\mathrm{LT}}$ (rapport $\mathrm{a}_{\mathrm{LTp}} / \mathrm{a}_{\mathrm{LT}}$ supérieur â 1 'unité); pour de tels angles, Tes variations de 1 anisotropie tissulaire $\left(\mathrm{E}_{\mathrm{L}}^{*} / \mathrm{E}_{\mathrm{T}}^{*}\right)$ sont donc majoritairement dues aux variations de l'orientation $(\varphi)$ des microfibrilles dans la sous couche $S_{2}$.

Dans le cas d'un tissu de bois final présentant à la fois une masse volumique élevée (épaisseurs de paroi importantes conduisant à un terme de flexion $\mathrm{a}_{\mathrm{F}}$ quasi nul), et une valeur de $(\varphi)$ inférieure ou égale à $10^{\circ}$, l'anisotropie élastique $\left(\mathrm{E}_{\mathrm{L}}^{*} / \mathrm{E}_{\mathrm{T}}^{*}\right) \mathrm{du}$ tissu ligneux résulte donc de l'anisotropie pariétale $\mathrm{a}_{\mathrm{LTp}}$ majoritairement pilotée par le paramètre $(\varphi)$.

A contrario, pour des masses volumiques tissulaires faibles et des angles $(\varphi)$ supérieurs à $10^{\circ}$, la relation d'ordre entre a et a s'inverse ; un tissu de bois initial présentera donc une anisotropie $\left(\mathrm{E}_{\mathrm{L}}^{*} / \mathrm{E}_{\mathrm{T}}^{*}\right)$ nettement plus sensible à la flexion des parois qu'un tissu de bois final. 


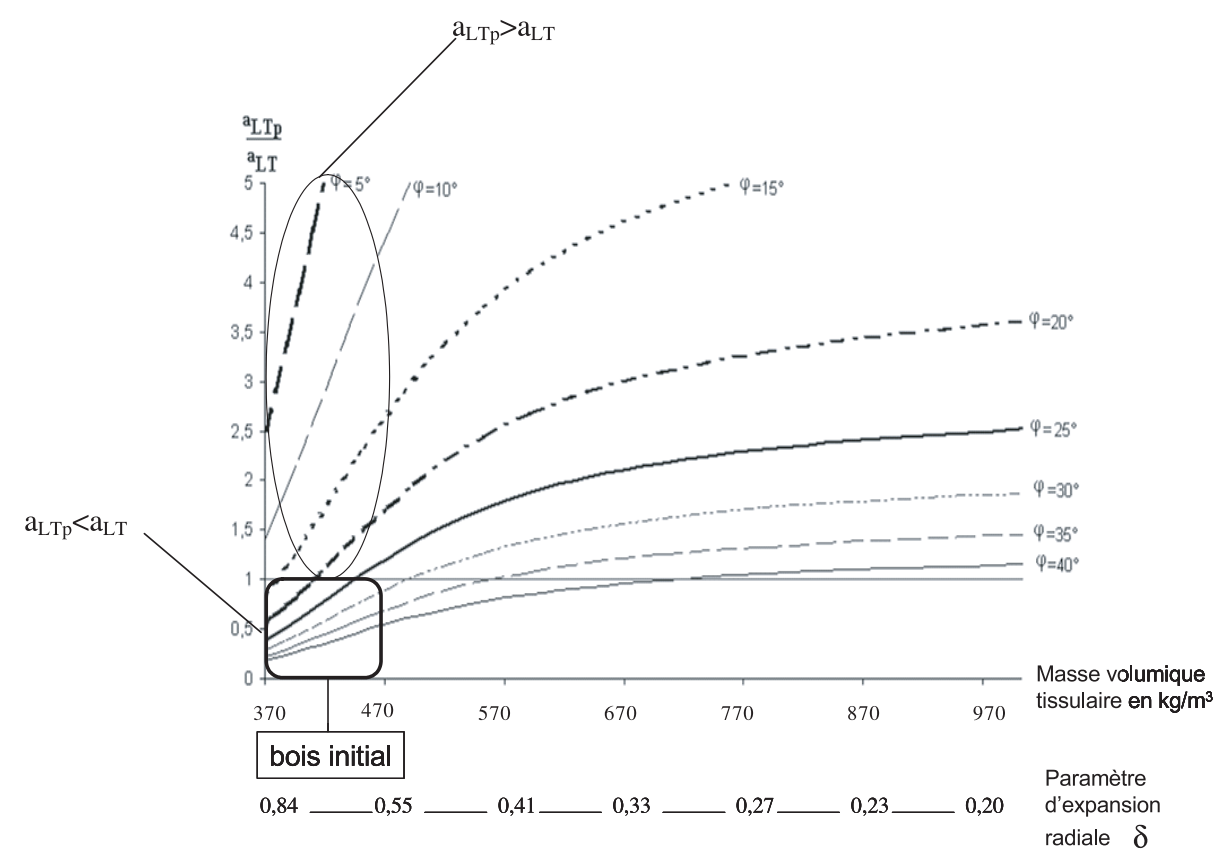

Figure 7. Relation d'ordre entre le rapport d'anisotropie élastique pariétal aLTp et le rapport d'anisotropie élastique a volumique tissulaire et de l'expansion radiale cellulaire.

Enfin, et en référence au résineux standard [10,12] évoqué précédemment, la figure 7 montre que pour un tissu de masse volumique $450 \mathrm{~kg} / \mathrm{m}^{3}$ présentant un angle compris entre $5^{\circ}$ et $25^{\circ}$, l'essentiel de l'anisotropie élastique $\left(\mathrm{E}_{\mathrm{L}}^{*} / \mathrm{E}_{\mathrm{T}}^{*}\right)$ est imputable à l'orientation des microfibrilles $(\varphi)$ dans la sous couche $S_{2}$.

\section{CONCLUSION}

L'anisotropie élastique macroscopique des résineux est souvent attribuée à l'influence d'une ou de plusieurs séries de paramètres structuraux. Par une hiérarchisation analytique de leur incidence, il est montré ici qu'elle est au premier chef la conséquence de l'anisotropie élastique de la double paroi principalement gouvernée par l'orientation $(\varphi)$ des microfibrilles de cellulose dans la sous couche pariétale $S_{2}$. A cette échelle de description, au moins deux options de modélisation, fortement complémentaires peuvent être envisagées :

- les modèles Multicouches qui, par leur approche du type « stratifié » reflètent assez fidèlement la structure pariétale « en couche » des résineux,

- un modèle voisin à squelette de fibres qui assimile quant à lui le renfort filamentaire cellulosique de la sous couche $\mathrm{S}_{2}$ à un treillis de microfibrilles entrecroisées.

Quelle que soit l'option de modélisation envisagée à l'échelle de la double cloison, l'anisotropie élastique tissulaire est fortement dépendante de l'anisotropie élastique de la double paroi [7]. On montre ici, dans le cas du modèle Squelette, que l'essentiel du comportement élastique d'un tissu de masse volumique $450 \mathrm{~kg} / \mathrm{m}^{3}$ présentant un angle $(\varphi)$ compris entre $5^{\circ}$ et $25^{\circ}$ découle des variations de l'orientation des microfibrilles dans la double cloison. Cette tendance est particulièrement marquée sur un tissu de bois final de forte masse volumique $\left(750 \mathrm{~kg} / \mathrm{m}^{3}\right)$ et un angle $(\varphi)$ compris entre $5^{\circ}$ et $10^{\circ}$. Pour un tissu de bois initial présentant de faibles épaisseurs pariétales et un $(\varphi)$ supérieur, le terme de flexion de paroi devient prépondérant dans l'explication du rapport $\left(\mathrm{E}_{\mathrm{L}}^{*} / \mathrm{E}_{\mathrm{T}}^{*}\right)$.

Plus généralement, le travail présenté ici propose une schématisation simplifiée, mais pragmatique, de la microstructure tissulaire, permettant de rendre compte du rôle joué par l'angle des microfibrilles sur l'anisotropie élastique Longitudinale/ Tangentielle du tissu ligneux. Sans remettre en cause la hiérarchisation démontrée ici, le réalisme biologique de certaines étapes intermédiaires (Passages) de la modélisation gigogne pourra sans doute être amélioré à l'avenir via l'utilisation de techniques complémentaires de caractérisation (analyse d'images et détermination de la forme de la cellule moyenne motif du milieu périodique, [17]) habituellement utilisées en sciences des matériaux.

\section{RÉFÉRENCES}

[1] Barrett J.D., Theorical models of wood shrinkage and elasticity, Ph.D. University of California, Berkeley, 1973.

[2] Bergander A., Local variability in chemical and physical properties of spruce wood fibers, Doctoral thesis, Stockholm, 2001.

[3] Bodig J., Jayne B.A., Mechanics of wood and wood composites, Van Nostrand Reinhold, New York, NY, 1982.

[4] Denne M.P., Definition of latewood according to Mork (1928), IAWA Bulletin n.s.10 (1988) 59-62.

[5] Erickson H.D., Harrisson A.T., Douglas Fir Wood Quality Studies, Part I: Effects of age and stimulated growth on wood density an anatomy, Wood Sci. Technol. 8 (1974) 207-225. 
[6] Gachet C., Guitard D., Gril J., The importance of S2 sub- layer in the description of cell wall anisotropy in softwood, Proceedings of the second International Conference of the European Society for Wood Mechanics, Stockholm, Sweden, May 25th-28th 2003, pp. 319-324.

[7] Gachet C., Inventaire et hiérarchisation de paramètres structuraux et ultrastructuraux facteurs de variabilité intra spécifique de certaines propriétés mécanophysiques des tissus ligneux, Thèse en Sciences du bois, Université Bordeaux 1, 2003.

[8] Gibson L.J., Ashby M.F., Cellular solids - Structure and properties, Pergamon Press, 1988.

[9] Gillis P.P., Orthotropic elastic constant of wood, Wood Sci. Technol. 6 (1972) 138-156.

[10] Guitard D., Mécanique du matériau bois et composites, Éditions Cépadues, 1987.

[11] Guitard D., Masse H., Yamamoto H., Okuyama T., Growth stress generation: a new mechanical model of the dimensional change of wood cells during maturation, J. Wood Sci. 45 (1999) 384-391.

[12] Guitard D., Gachet C., Paramètres structuraux et/ou ultra structuraux facteurs de variabilité intra arbre de l'anisotropie élastique du bois, Ann. For. Sci. 61 (2004) 129-139.

[13] Kollmann F.P., Cote W.A., Principles of wood sciences and technology: 1, Solid wood, Springer-Verlag, New York, 1984.

[14] Koponen S., Toratti T., Kanerva P., Modelling elastic and shrinkage properties of wood, Wood Sci. Technol. 23 (1989) 53-63.

[15] Koponen S., Toratti T., Kanerva P., Modelling longitudinal elastic and shrinkage of wood based on cell structure, Wood Sci. Technol. 25 (1991) 25-32.
[16] Marguerie D., Begin Y., Cournoyer L., Distinction anatomique du bois du Mélèze (Larix laricina [Du Roi] K. Koch), de l'épinette blanche (Picea glauca [Moench.] Voss), et de l'épinette noire (Picea mariana [Mill.] B.S.P.), en vue de l'analyse des macro restes, Géographie physique et Quaternaire 54 (2000) 317-325.

[17] Marion C., Caractérisation de l'agencement cellulaire du bois à l'aide de critères morphologiques mesurables par analyse d'images, rapport de DEA Sciences du Bois, ENGREF, Nancy, 2001.

[18] Mark R.E., Cell wall mechanics of tracheids, New Haven and London, Yale University Press, 1967.

[19] Navi P., Rastogi P.K., Gresse V., Tolou A., Micromechanics of wood subjected to axial tension, Wood Sci. Technol. 29 (1995) 411-429.

[20] Norimoto M., Takabe K., On noncrystalline structure of wood, Wood Res. Technical Notes, 21 (1985) 96-101.

[21] Perre P., Keller R., La prédiction des propriétés macroscopiques du matériau bois à partir de sa structure anatomique : besoin ou moyen de caractériser la paroi ? J. Trace Microprobe Tech. 12 (1994) 277288.

[22] Ruelle J., Anatomie comparative bois normal/bois de réaction et observation des relations structure/propriétés du bois de six espèces d'angiospermes de forêt tropicale humide et de trois espèces de gymnospermes de zone tempérée, rapport de DEA Sciences du Bois, INRA, 2003.

[23] Sakurada I., Nukushina Y., Ito T., Experimental determination of the elastic modulus of crystalline region in oriented polymers, J. Polymer Sci. 57 (1962) 651-660.

[24] Sahlberg U., Salmen L., Oscarsson A., The fibrillar orientation in the S2 layer of wood fibres as determined by X-ray diffraction analysis, Wood Sci. Technol. 31 (1997) 77-86. 